\title{
ABOUT ASSESSING THE LEARNING OF STUDENTS WITH DYSCALCULIA
}

\author{
María Isabel García-Planas ${ }^{1}$, María Victoria García-Camba Vives² \\ ${ }^{1}$ Universitat Politècnica de Catalunya (SPAIN) \\ ${ }^{2}$ Clínica Corachan (SPAIN)
}

\begin{abstract}
Dyscalculia is a neurodevelopmental disorder that affects to the capacity for calculation, interfering with the academic performance, the level of social inclusion, the access to the working world, and especially in the realization, both personal and professional of the people who suffer it. This learning disability is nearly as common as dyslexia, nevertheless, it is studied much less, and it is worse understood, and so many times it is diagnosed inconsistently.

With regard to academic performance, efforts are being made to improve learning, both at the level of the emerging scientific field of educational neuroscience and from the point of view of education. However, to date, scientists have only been studying how to help children affected by dyscalculia to develop new brain strategies that aim to improve their learning without taking into account the way to evaluate these learning.

Through the exploration of subjacent neurocognitive processes inherent to practice and educational theory, the main objective of this work is to analyse the possible difference in the brain function of affected individuals with dyscalculia in the performance of evaluation tests in order to find the best way to be evaluated, to intervene in the strengthening of arithmetic processing and to improve their performance and self-esteem.

The methodology used to achieve the desired goal is to analyse the activity of the brain during computation and answering test questions by using evoked potentials and to check with the dyscalculic students as they reasoned during the performing tests comparing them with students who do not have this learning difficulty.

After the analysis carried out, we concluded that to evaluate students with dyscalculia, the test type should be avoided and must use alternative evaluation methods such as the oral examination, while at the same time allowing certain actions that can help them. Due to the difficulty of learning about the people who suffer this difficulty, the probability of them responding poorly in this kind of evaluation, regardless of the knowledge of the subject it is very high, so the result of the test is not valid to assess your learning, which is the final objective of the test.
\end{abstract}

Keywords: Dyscalculia, brain, education, learning evaluation.

\section{INTRODUCTION}

It is well known that the evaluation process has a deep effect on student learning and its practices have severe repercussions on the learning process of each student and on the educational procedure in general.

Students with learning difficulties raise special educational needs, which must be given adequate educational responses of an extraordinary nature, different from those needed by most students. These responses are usually considered in the learning process, but must also be taken into account in the assessment of the learning of these students.

It is a teacher's responsibility to provide a suitably differentiated subject curriculum, accessible to all students, that provides each with the opportunity to develop and apply individual strengths. Responsibilities for meeting the additional needs of students with learning difficulties are the same as those for all students and should include approaches that avoid unnecessary exposition of the student on tasks where its specific difficulty prevents it from being carried out successfully.

Assessment is an essential component of the learning process whose purpose is to determine to what extent the quality goals established in the standards are being met, associated with the learning that students are expected to achieve during their formative stage. 
The evaluation offers possibilities to strengthen and consolidate the learning, as well as the achievements of the objectives or purposes in any field of study. The evaluation makes it possible to show which are the priority needs that must be met and must show congruence between knowledge and student performance.

From the Butterworth [1], studies, people with dyscalculia not only have difficulties in arithmetic but also with spatial orientation, control of their own actions and representation of their own body, especially the fingers. Also Ta'ir and Al. in [2], mention that they usually present visual, tactile and psychomotor skill problems. These data show that students affected by dyscalculia not only have difficulties with arithmetic operations but also with the performance of activities in which they need visual, tactile, psychomotor or spatial orientation skills.

These skills in one way or another intervene in the performance of exams, so it is essential to study how students with learning difficulties should be tested and especially those affected by dyscalculia.

The latest research on dyscalculia indicates that there are cognitive and psycholinguistic processes that develop in the preschool stage and that are decisive for the learning of calculus in children, that is why early detection is essential. There are better methods of early detection of the same that will allow to start an adequate early attention (for example [3], [4]). The sooner the dyscalculia is detected and the corrective measures begin to be applied, the fewer problems these students will have in front of the exams as their level of stress will decrease. Nevertheless, it is important to deepen in how students, with this learning difficulty, should be evaluated. Students with learning difficulties require modifications not only in the learning methodology but in the evaluation system.

\section{DYSCALCULIA DISORDER AND THE BRAIN}

Dyscalculia is a complex learning disorder that affects basic numerical processing and math learning. This disorder is much less studied than dyslexia although, has a prevalence of approximately the same number of people around $3-6 \%$ of schoolchildren.

The mathematical process is a very complicated multimodal neurocognitive function, which is in conjunction linked to other cognitive processes such as language, executive functioning, spatial structuring, and memory. Diverse areas of the human brain are involved in the computational capacity, conforming neural networks, for this reason, different lesions in the brain can produce disturbance on data processing, ([3], [5]).

In the following figure, Fig. 1, the brain areas related to mathematics are shown. Some areas involved in mathematical processing include motor cortices, somatosensory, visual and primary auditory.

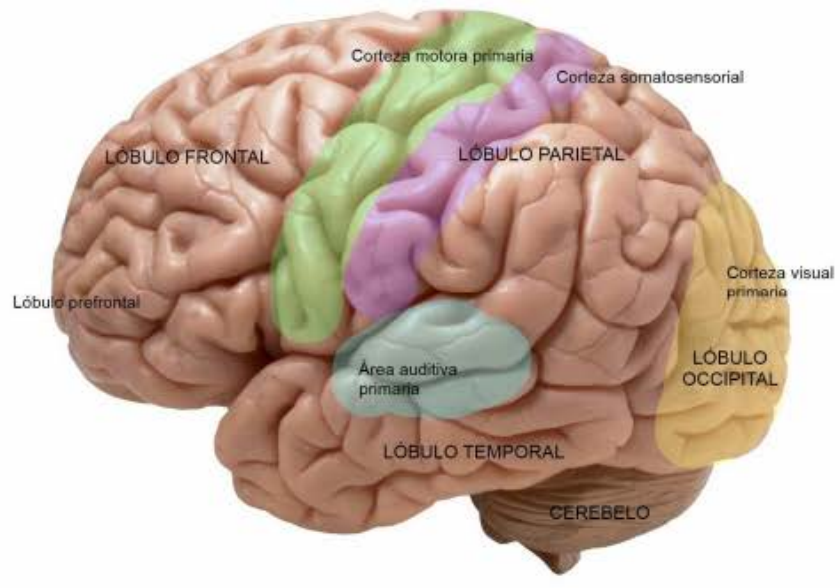

Figure 1 Brain Areas. From [6].

It is difficult to locate mathematical knowledge in the brain because different circuits intervene that can act in a partially autonomous way. Nevertheless, recent analyses of brain images show some differences in brain function and structure in people with dyscalculia. These differences are found in the surface area and in the thickness and volume of certain parts of the brain. There have also been found some differences in the activation of areas of the brain associated with numerical and mathematical processes. These areas are connected with essential learning skills, such as memory 
and planning. The images obtained indicate number processing activates interconnected number networks centered in both right and left parietal lobes, in particular the IPS region, with the left angular gyrus somewhat more involved in language-dependent driving of exact numbers and the right angular gyrus in continuous magnitude estimation, [7].

Another characteristic of people with dyscalculia is the difficulty to read numbers fluently. This difficulty is due to alterations in the occipitotemporal area of the brain. The occipitotemporal zone, also called area for the visual form of the elements, is considered the neural sign of dyscalculia and also of dyslexia since it has been proven that this area has low activation in people with this alteration

The figure Fig. 2, obtained by means a functional nuclear magnetic resonance, shows some areas of the brain that participate in the calculation

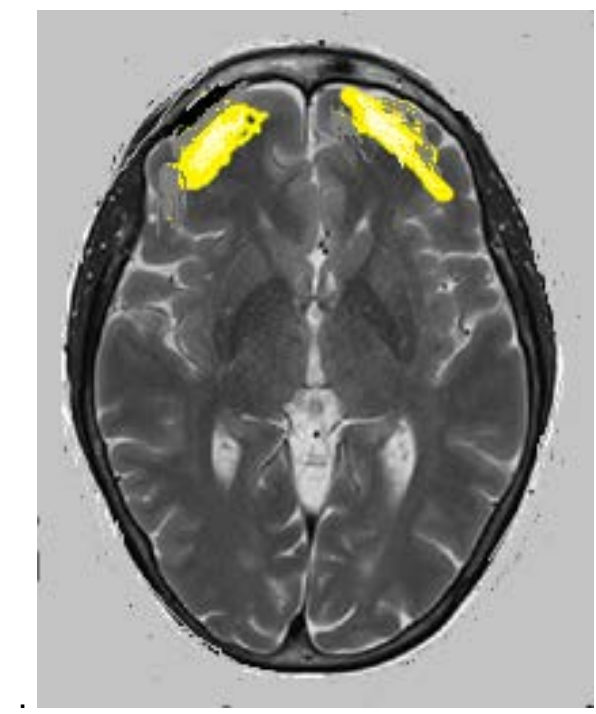

Figure 2. fRMN result, test performed by the authors

Guidelines of brain activity in 4-year-olds and adults evince overlapping areas in the parietal lobes bilaterally when responding to changes in numerosity [8]. However, there is a developmental path in the organization of more complex arithmetical skills.

The organization of routine numerical activity changes with age, altering from frontal areas and medial temporal areas to parietal areas and occipitotemporal areas [9].

These changes allow the brain to process numbers in a more efficiently and automatically manner, consequently, enables it to process more complex arithmetical calculations.

This suggests the possibility that the neural specialization for arithmetical processing may come up, at least in part, from a developmental interaction between the brain and experience ([10], [11]). Thus, one way of thinking about dyscalculia is that the typical school environment does not provide the right sort of experiences to enable the dyscalculic brain to develop normally to learn arithmetic.

The fact that, as indicated by Butterworth [1], the numerical abilities are generally correlated with the left parietal lobe, does not imply that other parts of the brain do not intervene. In fact, we have observed that when the brain performs cognitive activities, in normal subjects the beta wave appears (fast rhythms at 13-21 Hz) in the frontal lobes of the left hemisphere (see Fig. 3), and in children with learning problems, when performing cognitive tasks, the presence of abundant slow waves in the temporal lobes are observed. 


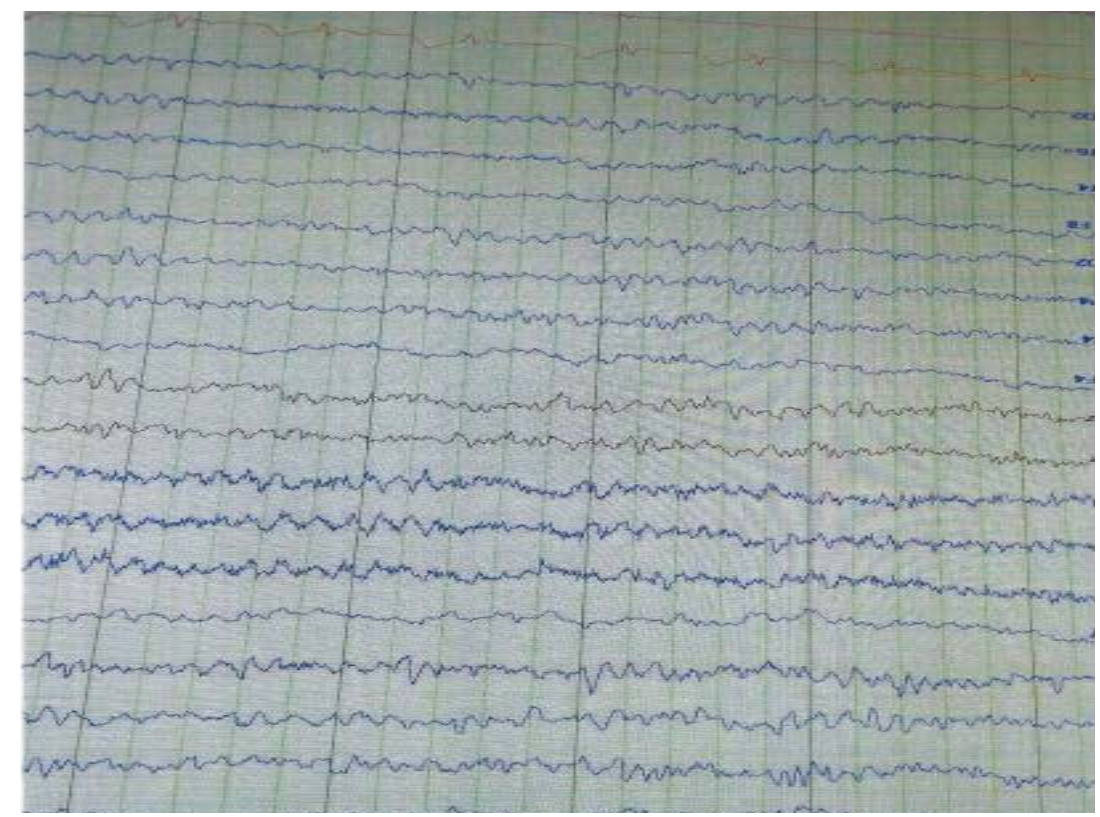

Figure 3. Electroencephalogram (EEG), test performed by the authors

On the other hand, in conducting tests using visual evoked potentials (VEP) it has been found that the parietal and occipital regions are the most affected in learning disorders since these areas, among other functions, are responsible for integrating the perception.

The amplitudes of the visual evoked potentials of the pattern type must be taken into account when understanding the mechanisms of visual processing and brain compensation in patients with dyslexia and dyscalculia.

Significant differences were observed for the amplitudes of the EPs in both monocular and binocular stimulation, with a $p$ less than 0.05 , without observing alterations in the latencies.

When performing cognitive activities, the beta waves $(13 \mathrm{~Hz}-21 \mathrm{~Hz})$ appear in the frontal lobe of the left hemisphere. There is a slow wave excess in the temporal lobes when performing cognitive tasks in children with learning problems.

The following figure Fig. 4, shows the graph of the visual evoked potential of a person with dyscalculia and a person without learning difficulties.

a)

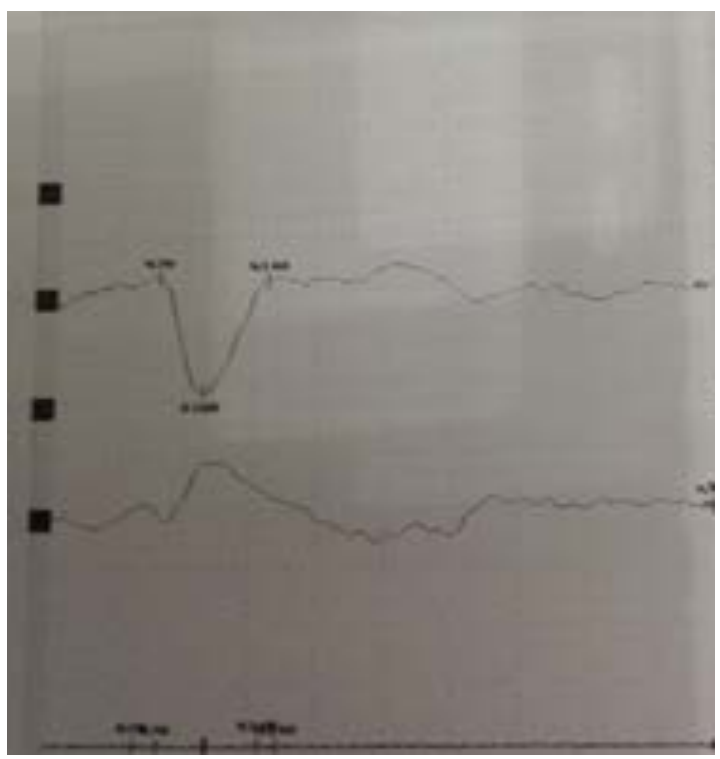

b)

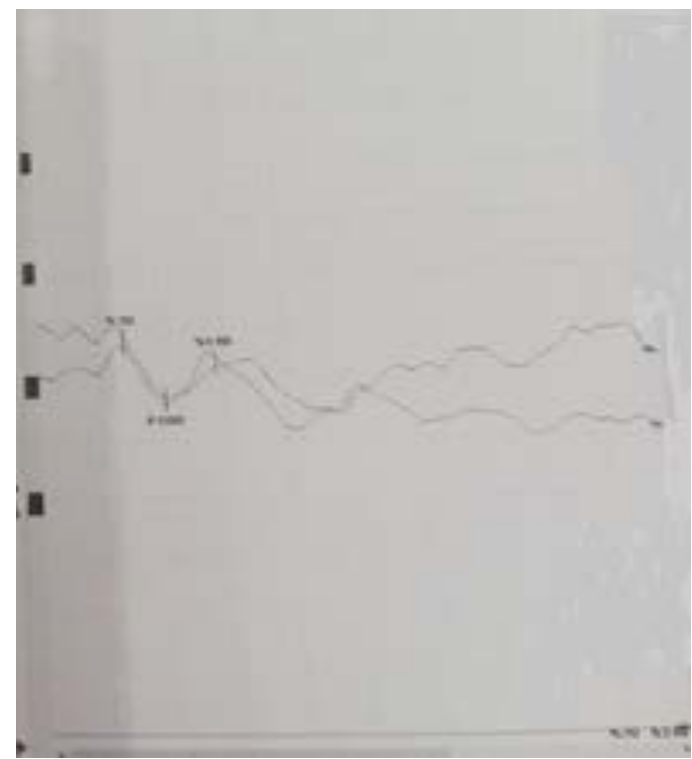

Figure 4.Visual Evoqued Potentials (VEP), a) dyscalculic, b) normal, tests performed by the authors 
We also want to emphasize that, although not all authors agree, for people with learning difficulties, there could also appear anomalies in the cerebellum responsible for the alterations in motor sequencing and in the acquisition of automatisms. In this sense, Nicolson, Fawcett, and Dean [12] proposed the hypothesis of the cerebellar deficit, according to which the automation of skills such as motor sequencing, articulation or reading is affected by the dysfunction of the cerebellum.

As Butterworth, et al. [13] say very well, an understanding of how the brain processes underlying number and arithmetic concepts will help focus teaching interventions on critical conceptual activities and will help focus neuroscience research on tracking the structural and functional changes that follow intervention.

\section{LEARNING ASSESSMENT FOR DYSCALCULIC STUDENTS}

To be able to analyze how exams should be prepared for students with dyscalculia, it is convenient to know their symptoms

\subsection{Symptoms of dyscalculia}

Dyscalculia is understood as a specific learning difficulty for mathematics or, more specifically, for arithmetic. The dyscalculia can generate, to the sufferer, different types of difficulties associated with mathematics and its characteristics and symptoms usually vary according to the age of the person, and it can already be detected in the pre-school stage.

People affected by dyscalculia present certain difficulties such as:

- Difficulty counting backwards

- Difficulty remembering 'basic' facts

- Slow to perform calculations

- Weak mental arithmetic skills.

- A poor sense of numbers \& estimation

- Difficulty in understanding place value

- Addition is often the default operation

- High levels of mathematics anxiety

Other difficulties no less important are:

- Difficulties in spatial organization

- Procedural difficulties

- Difficulties of judgment and reasoning

- Difficulties with mechanical memory

- Difficulties in numbering and calculation

- Orientation problem.

There are great differences in the activation of the occipitotemporal zone between the readers with development within the margins of normality and children with dyscalculia (and also with dyslexia), because while in the first cases there is strong connectivity between the brain word form area and Broca's area, which suggests that orthographic processing is integrated with the phonological, in people with this difficulty, instead, the connection occurs with the right frontal cortex that is associated with memory. This suggests that people with this disorder use different memory strategies to compensate for their difficulties in connecting orthographic and phonological information. Possibly, these strategies are intended to memorize the records because of the difficulty to establish connections between letters, numbers and sounds.

\subsection{Learning assessment}

The list of difficulties presents in the previous subsection, show that a student with dyscalculia may have difficulties in taking exams in subjects other than mathematics. Students who have dyscalculia symptoms face the exams with negative thoughts and with great anxiety as a result of fear of failure or 
pressure perceived in their environment to overcome different subjects, and especially in regard to the subject of mathematics. This is a type of execution anxiety, that appears in situations where the answer or the correct result of an activity is very important. Anxiety harms the concentration necessary for the good execution of the task.

Before deciding how the exam should be we must make it clear that we understand by evaluation. Following Serrano [14], the evaluation should be understood as an activity in which two dimensions are combined, one ethical and the other technical-methodological. The evaluation, besides having a technical aspect, no for that less complex, of the procedures and instruments and ways of obtaining more adequate information, poses, above all, an ethical problem, of deciding why to evaluate, for what and what to evaluate, with what legitimacy can one expect to evaluate. In this way, the technicalmethodological dimension becomes dependent on the ethical dimension. Only after having a clear sense of the evaluation in this area (for what and what to evaluate) can one consider what procedures (how to evaluate) could be useful for the purposes pursued.

In this point where the interest is to obtain the best manner to assess the students with dyscalculia, it cannot lose sight of the neuroscience that goes behind the principles of Universal Design for Learning [15]. These principles are related to the use of some areas of the brain during the learning process. In particular, for the assessment process, is important to consider the second principle corresponding to multiple means of expression, where it shows that each student has the capacity to express knowledge in a different way.

The authentic assessments for learning should stress the fact that student evaluations should help learners to understand and master the knowledge and skills that they receive through teaching, [16].

For the sake of finding the best way to evaluate the learning of students with dyscalculia, the first things we analyze are the characteristic features which affect them when performing an exam, among all characteristics the following should be highlighted:

- Commit transcription errors

- Confusion of similar graphics

- Problems of spatial orientation (confusion between left or right and between up and down)

- Count back

- Problems of position (confusion with the one above, below, with seriation and with reversibility).

These features affect very negatively in the performance of exams causing, in addition, great anxiety, blockage, and feeling of failure in people with dyscalculia when they face them. Other actions that can help students with dyscalculia in the performance of their exams are, to provide them more time to carry it out, that the exam is shorter, or allow to answer any of the questions orally. Depending on the age of the student (primary school, for example), it is convenient that the teacher provides the dates of the exam in advance, that the typography used for the test statement is clear and the size of the letter and line spacing is a little big than usual.

With regard to primary and secondary students, and referring to the rest of the students in the class, it is very important to let the classmates know what is the dyscalculia to avoid that they consider that the adaptations of the learning and its evaluation that realize for the affected student are not a privilege on him.

Once evaluated, the exam must be discussed with the student so that he can observe his mistakes and he can observe which have been as a result of his difficulty and so that the student can strengthen well the skills that allow him through specific exercises to overcome his difficulty.

These actions can also be useful for students with some other learning difficulty such as dyslexia.

In addition to these actions, it is important to carry out more specific actions for dyscalculic students to perform an exam in better conditions. In particular, it should allow:

- The use of the calculators and tables,

- To perform the exam on graph paper of $1 \mathrm{~cm}$ (in fact, between $0.6 \mathrm{~mm}$ and $1 \mathrm{~cm}$ depending on age)

- To use pens of two or more (depending on age) different colors

- Explicitly describe the formulas that are used 
- Allow the use of the emerging new technologies to solve numerical writing problems.

- Deliver the exam proposals in a format with increased font size and a greater line spacing.

Another important point to keep in mind is that people with dyscalculia have great difficulty answering multi-answer test questionnaires, in particular they have big problems with regard to inverted questions (in negative, ...), ambiguous questions, or questions with two or more, correct answers. As a consequence, examinations of this type should be avoided as much as possible, or carried out orally with direct questions.

In any case it is important that teachers are informed of the difficulties of the child and give them your support. To evaluate their progress providing them, alternative ways to demonstrate their knowledge.

\section{CONCLUSIONS}

The learning difficulties configure for those who suffer them, a wall for their integral development. This wall slows the pace of learning and prevents the scope of the competencies that make up the syllabuses. It is, therefore, necessary to find learning strategies and evaluation of these learning to get rid of these barriers.

In this article, we have finalized the study in the case of the difficulty of learning in mathematics by providing a little more information to the existing one, about brain activity in the numerical process and we have analysed how this activity can affect the performance of exams in students with dyscalculia.

After the analysis carried out, we concluded that to evaluate students with dyscalculia, the test type should be avoided, using alternative evaluation methods such as the oral examination, while at the same time allowing certain actions that can help them. Save the mistakes you usually make due to your difficulty. Due to the difficulty of learning of the people who suffer from this difficulty, the probability of them responding poorly, regardless of the knowledge of the subject it is very high, so the result of the test is not valid to assess your learning, which is the final objective of the test.

If we really want to break barriers we must not only adapt learning to the way of learning of students with learning difficulties but we must also adapt the way of evaluating them so that they can really show what they have learned.

\section{REFERENCES}

[1] B. Butterworth, The mathematical brain, Macmillan Publishers. London, United Kingdom, 1999.

[2] Ta'ir, J, Brezner, A. Ariel R. "Profound developmental dyscalculia: evidence for a cardinal/ordinal skills acquisition device." Brain and Cognition vol. 35, pp. 184-206, 1997.

[3] García Planas, M. I., \& García-Camba Vives, M. V. "Dyscalculia, mind, calculating brain and education". In EDULEARN18: 10th Annual International Conference on Education and New Learning Technologies, Proceedings book, pp. 0480-0489, 2018.

[4] Domínguez García, M.I. "Elaboración de un Test para la detección de riesgo de discalculia para uso de docentes". Adaya Press. 2019.

[5] J.A. Portellano, Neuropsicología infantil. Madrid, España Síntesis; 2008.

[6] R. A. Vargas Vargas. "Matemáticas y neurociencias: una aproximación al desarrollo del pensamiento matemático desde una perspectiva biológica" Revista Iberoamericana de educación matemática, vol. 36. pp. 37-46, 2013.

[7] I. Rapin "Dyscalculia and the Calculating Brain" Pediatric Neurology, vol. 61, pp. 11-20, 2016.

[8] J. F. Cantlon, E. M. Brannon, E. J. Carter, K. A. Pelphrey, PLoS Biol. vol. 4, e125, 2006.

[9] D. Ansari, "Effects of development and enculturation on number representation in the brain", Nature Reviews Neuroscience, vol. 9, no 4, pp. 278-291, 2008

[10] D. Ansari, A. Karmiloff-Smith, "Atypical trajectories of number development: A neuroconstructivist perspective". Trends in cognitive sciences, vol, 6 no 12, pp. 511-516, 2002.

[11] M. H. Johnson, " Functional brain development in humans" Nature Reviews Neuroscience. vol. 2, no 7, pp. 475-483, 2001. 
[12] R. I. Nicolson, A. J. Fawcett, P. Dean. "Developmental dyslexia: The cerebellar deficit hypothesis" Trends in Neurosciences vol.24, no 9, pp. 508-511, 2001.

[13] B. Butterworth,V. Sashank, D, Laurillard, "Dyscalculia: From Brain to Education", Science, vol. 332, pp. 1049-1053, 2011.

[14] S. Serrano de Moreno, "La evaluación del aprendizaje: dimensiones y prácticas innovadoras,"

[15] Educere, vol. 6, no. 19, pp. 247-257, 2002.

[16] B. Butler, "What does Multiple Intelligences theory and practice have to offer universal design for learning?" in proceedings of Is Universal Design in Educational any of my Business? AHEAD Educational Press, pp. 17-32, 2013

[17] L. Mundia, "The Assessment of Math Learning Difficulties in a Primary Grade-4 Child with High Support Needs: Mixed Methods Approach," International Electronic Journal of Elementary Education, vol. 4, no. 2, pp. 347-366, 2012. 